\title{
Diacronie
}

Studi di Storia Contemporanea

$\mathrm{N}^{\circ} 28,4 \mid 2016$

La voce del silenzio

\section{Emily K. Brock, Money Trees. The Douglas Fir and American Forestry, 1900-1944}

\section{Swen Steinberg}

Traduttore: Alessandro Salvador

\section{OpenEdition}

\section{Journals}

\section{Edizione digitale}

URL: http://journals.openedition.org/diacronie/4638

DOI: $10.4000 /$ diacronie.4638

ISSN: 2038-0925

\section{Editore}

Association culturelle Diacronie

Notizia bibliografica digitale

Swen Steinberg, «Emily K. Brock, Money Trees. The Douglas Fir and American Forestry, 1900-1944»,

Diacronie [Online], № 28, 4 | 2016, Messo online il 29 décembre 2016, consultato il 24 septembre 2020.

URL : http://journals.openedition.org/diacronie/4638; DOI : https://doi.org/10.4000/diacronie.4638 


\title{
Diacronie
}

N. 28 | 4|2016 La voce del silenzio: intelligence, spionaggio e conflitto nel XX secolo

\section{4/}

\section{PANORAMICA: Usa 2015}

\author{
Swen STEINBERG, Elisa GRANDI *
}

\author{
BURKE, Peter, What is the history of knowledge?, Cambridge-Malden, \\ Polity Press, 2015, 160 pp. \\ a cura di Swen STEINBERG \\ traduzione di Alessandro SALVADOR
}

Peter Burke, che con la sua opera Social History of Knowledge ${ }^{1}$, ha, in passato, offerto contributi essenziali per riorientare in termini storiografici le scienze umane e sociali, offre, con questo lavoro introduttivo, molto più che semplici approcci e metodi per la ancora giovane storia della conoscenza. Egli ne indica anche le fondamenta e i suoi sviluppi storici, che sono connessi a sociologi come Max Weber o Karl Mannheim e con gli approcci alla sociologia della conoscenza. La focalizzazione della storia della conoscenza, che prende in considerazione specifici patrimoni, i loro sviluppi, le distribuzioni e le modifiche, ricevette impulsi fondamentali dai dibattiti, occorsi negli anni Novanta, sulle moderne società del sapere e prende le distanze da una storia della conoscenza classica, orientata alle istituzioni, e dalla storia intellettuale, concentrata sulle persone. Dopo una breve introduzione sulla concettualizzazione storiografica della conoscenza, Burke presenta nel secondo capitolo i concetti principali: l'autorità e il monopolio della conoscenza, l'innovazione e l'interdisciplinarietà, gli ordini e le pratiche della conoscenza, il sapere situazionale ed estorto e, infine, il regime dell'ignoranza negli sviluppi consecutivi della conoscenza. Nel capitolo tre, Burke presenta le forme del sapere, differenziando le conoscenze collettanee (gathering knowledges), le conoscenze analitiche (analysing knowledges), le conoscenze diffuse

\footnotetext{
${ }^{1}$ BURKE, Peter, A social history of knowledge: from Gutenberg to Diderot, Cambridge-Malden, Polity Press - Blackwell Publishers, 2000.
} 
(disseminating knowledges) e le conoscenze applicative (employing knowledges). Queste forme del sapere vengono quindi collocate in una prospettiva storica ossia fatte risalire ad essa. Nell'ultimo capitolo vengono trattati, quindi, i problemi e le prospettive della storia della conoscenza. Una cronologia e una bibliografia essenziale completano un volume molto leggibile e semplice da consultare grazie all'indice analitico. Burke argomenta infine in modo convincente che, in una prospettiva a lungo termine, una storia della conoscenza può offrire nuove strade in ottica globale o transnazionale fintanto che vengano prese in considerazione le conoscenze in movimento (knowledge on the mover) o che ci si focalizzi sui produttori della conoscenza al di là della scienza $\mathrm{e}$ con l'aiuto di categorie moderne (ad esempio gli aspetti di genere).

\section{BOYER, Christopher R., Political Landscapes: Forests, Conservation, and Community in Mexico, Durham, Duke University Press, 2015, 360 pp.} a cura di Swen STEINBERG traduzione di Alessandro SALVADOR

L'analisi di Christopher R. Boyer offre un contributo cruciale sulla storia ambientale dell'America Latina e su tematiche che vanno oltre ai centri e verso le periferie, come era già accaduto con la prospettiva post-coloniale in riferimento all'India o all'Africa. In modo non dissimile da questi lavori, Boyer non si limita a prendere in considerazione interrogativi di tipo economico o scientifico collegati alle scienze forestali e ai dibattiti sulla conservazione. Egli va oltre e riscontra ioni sociali. Racconta così la sua storia ambientale come storia sociale, prendendo ad esempio le regioni del Michoacàn e del Chihuahua. La storia della moderna scienza forestale in queste regioni viene rappresentata come un costante conflitto tra il governo, gli studiosi delle foreste, le aziende di legname (in parte straniere) e la popolazione locale. Le foreste del Messico, la cui estensione si è ridotta di quasi il 50\% nel corso del ventesimo secolo, diventano quindi "panorami politici" poiché in esse si risolvono le dispute sull'utilizzo della natura o vengono fomentati conflitti tramite interventi esterni. Questo sguardo sull'agire e sul sapere di attori regionali viene contestualizzato nello sviluppo delle politiche forestali che, in Messico, hanno avuto una tendenza soprattutto liberale fino alla Seconda guerra mondiale per essere poi fortemente regolate dallo stato e approdare poi, negli anni Ottanta, ad una fase neoliberale.

2 BURKE, Peter, What is the history of knowledge?, Cambridge-Malden, Polity Press Blackwell Publishers, 2015, p. 123. 
BERRY, Evan, Devoted to Nature: The Religious Roots of American Environmentalism, Oakland, University of California Press, 2015, 272 pp. a cura di Swen STEINBERG

traduzione di Alessandro SALVADOR

Lo studio di Evan Barry congiunge la ricerca sulla storia ambientale con degli interrogativi sul mondo della religione e analizza i collegamenti tra l'ambientalismo americano e la religione tra Gilded Age e l'era progressista (ca. 1877-1920) andando però, alle sue origini e, in prospettiva, ben oltre questi confini. Berry si occupa di una tematica centrale nell'autocoscienza e nell'identità degli Stati Uniti quando erano ancora un giovane stato nazionale, in cui i legami con la natura e il concetto di luogo selvaggio giocavano inizialmente - e giocano in parte anche tuttora - un ruolo fondamentale. Anche se l'influenza che personalità legate al trascendentalismo come Ralph Waldo Emerson, Margaret Fuller o Henry David Thoreau hanno esercitato su questi concetti è stata sufficientemente studiata, le fondamenta teologiche e gli sviluppi successivi di stampo religioso non sono state finora sufficientemente analizzate. Evan Berry può quindi concludere che l'ambientalismo americano, anche nel Ventesimo secolo, è stato fortemente influenzato da elementi religiosi e che la sua comprensione della natura si è identificata soprattutto tramite il concetto cristiano della redenzione. Questo ha portato non solo ad un forte legame identitario con la natura, ma ha anche delineato gli approcci per definire cosa sia possibile fare all'interno della natura e come essa si possa utilizzare in senso turistico o ricreativo. I collegamenti illustrati da Berry mostrano quindi il potenziale dell'analisi storico-culturale superando le cornici interpretative usuali per il XIX e XX secolo, come lo stato nazionale, e indicano applicazioni che vanno al di là del contesto degli Stati Uniti.

BROCK, Emily K., Money Trees. The Douglas Fir and American Forestry, 190o-1944, Corvallis (Oregon), Oregon State University Press, 2015, 272 pp.

a cura di Swen STEINBERG

traduzione di Alessandro SALVADOR

Il lavoro di Emily K. Brock analizza lo sviluppo delle moderne scienze forestali prendendo ad esempio il patrimonio forestale degli stati dell'Oregon e di Washington, negli USA: Brock illustra i dibattiti scientifici ed ecologici come anche le prese di posizione delle industrie del legname e le strategie delle amministrazioni statali, con le 
relative implicazioni politiche. Il nodo centrale è che attorno al 1900, contemporaneamente alla crescita del movimento conservazionista, non solo si ebbe la professionalizzazione delle scienze forestali negli USA ma, a nord-ovest, si crearono delle vere e proprie Tree Farms nei vasti possedimenti del "barone del legno" Frederick Weyerahaeuser, in cui venivano piantati e fatti crescere rapidamente e in modo industriale gli abeti di Douglas. Questa economizzazione dell'ecologia, trattata spesso in modo sbrigativo nei trattati sul movimento conservazionista americano, si occupava anche della questione centrale di definire concetti come sostenibilità e senso civico. In seguito, la definizione della funzione che le foreste rivestivano per l'economia e la società cambiò, ma si sviluppò anche attraverso i metodi delle Tree Farms in un'ottica che privilegiava l'economia. Brock non riesce sempre a descrivere i diversi gruppi di attori nelle loro relazioni reciproche e anche il concetto di forestry rimane parzialmente indefinito nel suo utilizzo (scienza forestale, economia forestale o gestione delle foreste). Il lavoro di Emily K. Brock mostra però in modo convincente il potenziale di una ricerca focalizzata e centrata sugli attori che, combinata con i dibattiti scientifici, economici e pubblici, offre un contributo essenziale alla storia ambientale che, in una prospettiva comparativa, può trovare applicazione per altre regioni del mondo.

\section{PARSONS, Elaine Frantz, Ku-Klux The Birth of the Klan during Reconstruction, Chapel Hill (North Carolina), The University Of North Carolina Press, 2015, 272 pp.}

a cura di Elisa GRANDI

Il libro di Elaine Frantz Parsons, professoressa associata della Duquesne University (Pittsburgh, Pennsylvania) fornisce il primo resoconto dettagliato delle origini e dell'affermazione del Ku Klux Klan all'indomani della Guerra Civile. A differenza di altri episodi di violenza razziale che caratterizzarono gli anni immediatamente successivi alla sconfitta degli Stati del Sud, il Klan si affermò come un fenomeno radicalmente diverso, poggiando allo stesso tempo su di un forte radicamento locale e su di una visibilità nazionale. Dosando sapientemente un livello di dettaglio nella ricostruzione degli avvenimenti mai raggiunto nella storiografia sul tema e un'analisi dell'affermazione del discorso e dell'ideologia che accompagnò l'ascesa del Klan, l'autrice si muove su due livelli di analisi: da un lato lo studio degli "uomini che agivano sul campo", attraverso un insieme di relazioni politiche e comunitarie radicate nel territorio in cui operavano, dall'altro "l'idea del Klan" nel discorso pubblico, che viene ricostruita principalmente attraverso la stampa, locale e nazionale. Questa doppia 
prospettiva permette di dimostrare uno degli aspetti fondanti dell'analisi: la congiuntura di interessi tra bianchi del Sud e del Nord, tanto Repubblicani quanto Democratici, che permisero l'affermazione del Klan. Gli interessi rappresentati dai bianchi degli Stati del Nord amplificarono in maniera determinante il fenomeno del Ku Klux Klan, gli diedero una forma specifica permettendo che acquisisse una coerenza nazionale. 


\section{* Gli autori}

Swen Steinberg, storico, è professore associato in storia regionale alla Technische Universität di Dresda. Da ottobre 2014 fino a ottobre 2016 è ricercatore ospite presso la University of California Los Angeles con un finanziamento post-dottorale della Deutsche Forschungsgemeinschaft sul tema: Una storia transnazionale della conoscenza. Montagne, foreste e miniere tra Europa e America, 1760-196o. I suoi interessi di ricerca sono le concezioni dell'Economia del cultura, le trasformazioni economiche, la storia della conoscenza e gli studi sugli esuli. Ha pubblicato diversi libri e articoli su questi temi. Attualmente sta lavorando ad una analisi delle reti di giornalisti tedeschi esiliati in Cecoslovacchia (per «Metropol», «Berlin») e all'edizione dei carteggi di Kurt Eisner.

URL: < http://www.studistorici.com/progett/autori/\#Steinberg >

Elisa Grandi si è laureata in Storia all'Università di Bologna nel 2005, ha ottenuto la specializzazione in Storia del Mondo contemporaneo nello stesso ateneo, nel 2007. È alumna del Collegio Superiore dell'Università di Bologna. È stata Visiting Student alla Duke University (2009), Visiting Scholar alla New School of Social Research (2010), borsista Eiffel-Excellence presso il laboratorio SEDET di Parigi (2011) e Attaché de Recherche all'Université Paris Diderot (2011-2012). Sta completando un dottorato di storia economica all'Universita Paris Diderot, in cotutela con l'Università di Bologna, sui piani di sviluppo finanziati dalla Banca Mondiale in Europa e America Latina negli anni Cinquanta e Sessanta. Dal 2012 lavora come Senior Researcher nell'Equipex DFIH (Données Financières Historiques) della Paris School of Economics e dal 2014 insegna Storia della globalizzazione all'Université Paris 7 - Denis Diderot.

URL: < http://www.studistorici.com/2010/12/07/elisa_grandi/ >

\section{Per citare questo articolo:}

STEINBERG, Swen, GRANDI, Elisa, «Panoramica: Usa 2015», Diacronie. Studi di Storia Contemporanea : La voce del silenzio: intelligence, spionaggio e conflitto nel XX secolo, 29/12/2016,

URL:< http://www.studistorici.com/2016/12/29/usa_numero_28/ >

Diacronie Studi di Storia Contemporanea 0 www.diacronie.it

Risorsa digitale indipendente a carattere storiografico. Uscita trimestrale. redazione.diacronie@hotmail.it

Comitato di redazione: Jacopo Bassi - Luca Bufarale - Antonio César Moreno Cantano - Deborah Paci - Fausto Pietrancosta - Alessandro Salvador - Matteo Tomasoni - Luca Zuccolo

Diritti: gli articoli di Diacronie. Studi di Storia Contemporanea sono pubblicati sotto licenza Creative Commons 3.0. Possono essere riprodotti e modificati a patto di indicare eventuali modifiche dei contenuti, di riconoscere la paternità dell'opera e di condividerla allo stesso modo. La citazione di estratti è comunque sempre autorizzata, nei limiti previsti dalla legge. 